\title{
SARS-CoV-2 infection after vaccination in Italian health care workers: a case report
}

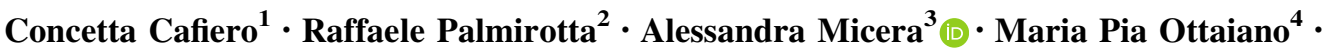 \\ Agnese $\mathrm{Re}^{5} \cdot$ Florinda Pedata $^{4} \cdot$ Domenico Costagliola $^{4} \cdot$ Domenico Ponticelli $^{6}$. \\ Salvatore Pisconti ${ }^{1} \cdot$ Beniamino Schiavone $^{6} \cdot$ Giulio Benincasa $^{4}$
}

Received: 7 October 2021/Revised: 27 November 2021/ Accepted: 25 January 2022/Published online: 6 March 2022

(C) The Author(s) 2022

\begin{abstract}
Following the approval of COVID-19 vaccination program by EMA and national authorities, an immunization campaign started in Italy with BNT162b2mRNA vaccine, initially focused on healthcare workers. The active immunization was monitored by systemic antibody titration and continuous surveillance was guaranteed by antigenic/molecular tests on swabs. Cases of infection have been recently observed in vaccinated healthcare workers. Herein we describe an outbreak of infection occurring in five physicians out of 656 healthcare workers belonging to a private hospital, referring mild symptoms of COVID-19. Healthcare workers underwent complete vaccination and screening for antibody titration. Five out of 656 healthcare workers were tested positive for SARS-CoV-2 in nasopharyngeal swabs and referred mild COVID-19
\end{abstract}

Concetta Cafiero, Raffaele Palmirotta have contributed equally to this work.

\footnotetext{
Raffaele Palmirotta

raffaelepalmirotta@gmail.com

$\bowtie$ Alessandra Micera

alessandra.micera@fondazionebietti.it

Medical Oncology, SG Moscati Hospital, Taranto, Italy

2 Interdisciplinary Department of Medicine, School of Medicine, University of Bari 'Aldo Moro', Piazza G. Cesare, 11, 70124 Bari, Italy

3 Research Laboratories in Ophthalmology, IRCCS Fondazione Bietti, via Santo Stefano Rotondo 6, 00184 Rome, Italy

4 Department of Clinical Pathology and Molecular Biology, Pineta Grande Hospital Castel Volturno (CE), Castel Volturno (CE), Italy

5 Catholic University of Sacred Heart, Rome, Italy

6 Pineta Grande Hospital, Castel Volturno (CE), Italy
}

symptoms. Molecular analyses were carried out to identify possible variants of Spike protein. Their genotyping performed on RNA extracts highlighted the presence of del69/ 70, N501Y, A570D, and 1841A > G (D614G) sequence variants, all indicative of VOC 202012/01-lineage B.1.1.7, suggesting a common source of infection. These cases might represent a serious emergency because outbreaks can compromise frail patients with important concomitant diseases.

Keywords Post-vaccinal infection · COVID-19 . Healthcare workers · Genetic variants

\section{Significant statement}

The risk of infection occurs even for vaccinated healthcare workers. Cases occurring in healthcare workers can represent a serious emergency because outbreaks can compromise frail patients and elder population. Considering the recently identified new variants, it is essential to genotype the cases of infection outbreaks. These findings have implications for public health strategies aiming to increase the level of community control and protection.

\section{Introduction}

The SARS-CoV-2 appeared first in Wuhan (China; Dec 2019) and quickly spread worldwide convincing the WHO to declare a COVID-19 pandemic (March 2020) [1, 2]. COVID-19 pandemic has caused 176.945.596 cases with 3.836 .828 deaths and particularly 3.4 million cases and 127,153 deaths in Italy (June 2021; ourworldindata.org). After the European Medicines Agency (EMA) 
recommendations (Dec 21, 2020), the European Commission authorized the first vaccine against COVID-19 based on BNT162b2 mRNA (Comirnaty: Pfizer and BioNTech) [3]. Quickly, the Italian Medicines Agency (AIFA) authorized a national immunization campaign (Dec 22, 2020) starting with healthcare workers, excluding those healed from SARS-CoV-2 disease [3]. Clinical trials for BNT162b2 vaccine estimated an early immunization efficacy of $52.4 \%$ at first dose and $90.5 \%$ at 7 days after two doses in preventing COVID-19 [4]. A recent study estimated a 92\% BNT162b2 effectiveness at 7 days after second dose (94\% symptomatic COVID-19, 87\% hospitalization, and 92\% severe COVID-19) [5]. Despite this high efficacy and effectiveness of vaccination campaign, cases of SARS-CoV-2 infection still occur in vaccinated population [5].

Herein, we describe an outbreak of infection in healthcare workers receiving two doses of BNT162b2 vaccine.

\section{Methods}

This study was carried out at the Pineta Grande Hospital (Castel, Volturno, Caserta, Italy). The intramural vaccination campaign started on Jan 30 (2021) and included the first dose of BNT162b2 mRNA vaccine followed by a second one after 21 days (Comirnaty, BioNTech-Pfizer, Mainz, Germany/New York, United States). Healthcare workers $(656 ; 353 \mathrm{~F} / 303 \mathrm{M})$ were asked to join the study by signing the informed consent (prot n. R.CE 30/21; Azienda Sanitaria Locale Brindisi Ethical Committee, Brindisi, Italy). The study was conducted in accordance with the International Council of guidelines for good clinical practice of harmonization and the regulations of the Italian State, following the principles of Declaration of Helsinki. All specimen collections, specific extractions, and analysis were carried out according to COVID-19 safety instructions. As routine, healthcare workers were subjected to i. analysis of total $\mathrm{IgG}$ raised against the S1-RBD antigen and ii. nasopharyngeal swab tests made every 30 days. AntiSARS-CoV-2 IgG quantification was performed by using the SARS-CoV-2 total chemiluminescent assay kit (COV2T) operating on the Advia Centaur XP System platform (Siemens Healthineers, Milan, Italy). Swabs were processed in an automated extraction platform (MagCore ${ }^{\circledR}$, RBC Bioscience Corp., New Taipei City, Taiwan) and total RNA was quantified using Qubit Fluorometer (Life Technologies, Carlsbad, CA, USA). Real time-qPCR was carried out using the AMPLI-SARS-CoV-2 commercial kits (Dia-Chem srl, Napoli, Italy) targeting the virus nucleocapsid $\mathrm{N} 1 / \mathrm{N} 2$ genes and the internal control RP gene, in a Real-Time PCR System (CFX 96; Bio-Rad, Hercules, CA, USA).
The genotyping was first performed by using AMPLI SARS-CoV-2 Variants commercial kit efficient for identifying 23 variants of Spike protein (Dia-Chem srl, Napoli, Italy). For variants' confirmation, a direct sequencing analysis was performed. PCR primers were designed using Genomics Expression DNA Sequence Analysis Software referring to CoV-2 Spike glycoprotein (GenBank: NC_045512.2). Amplicons were purified (PCR Purification Kit; EuroClone ExoStar PCR-plate) and directly sequenced on both strands using Big Dye Terminator V3.1 Cycle Sequencing kit on an ABI3500 Genetic Analyzer (Applied Biosystems, Foster City, USA).

\section{Results}

After vaccination, all healthcare workers were periodically monitored for specific virus immunoglobulins titration and subjected to molecular test for SARS-CoV-2 RNA expression every 30 days. This protocol was used for the purpose of identify asymptomatic or mild symptomatic SARS-CoV-2 positive subjects and assure a huge protection for healthcare personnel and patients.

Despite vaccination, five physicians were found positive to SARS-CoV-2 virus after 12 weeks from second dose, as detected by Real-Time PCR performed on RNA extracted from nasopharyngeal swab. The characteristics of these 5 healthcare workers are summarized in Table 1. Clinical assessment defined mild symptomatic SARS-CoV-2 positive subjects, according to a recommended schedule. Symptoms ranged from diarrhea (case 1), conjunctivitis (cases 2 and 5), muscle pain (cases 2, 4, and 5), breathlessness, fever higher than 38 (case 5) and lack of smells/tastes (case 3). All subjects had no concomitant diseases except for pre-obesity (cases 1,2, and 3) and obesity (cases 4 and 5) (Table 1).

The genotyping performed first by Real-time PCR and then confirmed by direct sequencing proved the presence of del69/70, N501Y, A570D, and 1841A > G (D614G) variants, indicative of VOC 202,012/01-lineage B.1.1.7 [6], in all samples suggesting a common source of infection (Fig. 1).

Despite an epidemiologic investigation conducted by the hospital, neither the source nor modality of SARS-CoV-2 infection could be identified.

\section{Discussion}

The development of COVID-19 disease was significantly reduced by worldwide vaccination campaign, although several recent studies highlighted the presence of outbreaks of infection among fully vaccinated healthcare workers. 
Table 1 Laboratory, vaccination, and clinical data of healthcare workers found positive for SARS-CoV-2

\begin{tabular}{|c|c|c|c|c|c|}
\hline Case & 1 & 2 & 3 & 4 & 5 \\
\hline \multicolumn{6}{|l|}{ Demographic data } \\
\hline Age & 48 & 22 & 25 & 63 & 56 \\
\hline Sex & M & $\mathrm{F}$ & $\mathrm{F}$ & M & $\mathrm{F}$ \\
\hline BMI & 28.08 & 28.68 & 28.59 & 33.59 & 35.15 \\
\hline \multicolumn{6}{|l|}{ BNT162b2 mRNA vaccine ${ }^{a}$} \\
\hline dose I & $01 / 04 / 2021$ & $01 / 07 / 2021$ & $01 / 04 / 2021$ & $01 / 07 / 2021$ & $01 / 07 / 2021$ \\
\hline dose II & $01 / 25 / 2021$ & $02 / 04 / 2021$ & $02 / 01 / 2021$ & $01 / 28 / 2021$ & $01 / 28 / 2021$ \\
\hline \multicolumn{6}{|l|}{ Antigenic test $(A U / m L)$} \\
\hline $\operatorname{IgG}, 15$ days after dose $\mathrm{I}$ & 239.7 & 726 & 155.6 & 407 & 726.5 \\
\hline IgG, 20 days after dose II & 105 & 197.7 & 83.6 & 112 & 197.7 \\
\hline \multicolumn{6}{|l|}{ Real-time PCR (Cts) } \\
\hline \multicolumn{6}{|l|}{ At the time of infection } \\
\hline \multicolumn{6}{|l|}{3 months after dose I } \\
\hline N1 & 22.0 & 24.6 & 15.7 & 26.2 & 23.6 \\
\hline $\mathrm{N} 2$ & 20.0 & 23.7 & 16.9 & 27.3 & 24.4 \\
\hline \multicolumn{6}{|l|}{ Clinical symptoms } \\
\hline Fever & - & - & - & - & + \\
\hline Cold & - & - & + & - & - \\
\hline Conjunctivitis & - & + & - & - & + \\
\hline Mild cough & - & - & - & + & + \\
\hline Asthenia & + & - & - & - & - \\
\hline Diarrhea & + & - & - & - & - \\
\hline Headache & + & + & - & - & - \\
\hline Muscle pain & - & + & - & + & + \\
\hline Lack of smell/taste & - & - & + & - & - \\
\hline Dizziness and breathlessness & - & - & + & - & - \\
\hline
\end{tabular}

$M$, male; $F$, female; BMI Body Max Index; $C t s$ cycle threshold

${ }^{a}$ On January 2021, the hospital began the immunization campaign for 656 healthcare workers using the BNT162b2 mRNA vaccine (Comirnaty, BioNTech-Pfizer). Healthcare workers received the 2nd dose after 21 or 28 days and the five subjects resulted COVID-19 positive after 3 months after vaccination

Amit and coworkers (Jan 2021) reported a positivity rate of $0.77 \%$ at $1-14$ days and of $0.36 \%$ at $15-28$ days after the first dose in Israeli healthcare workers immunized with BNT162b2 COVID-19 vaccine [6]. Merely, 22 out of 4,081 vaccinated healthcare workers $(0.54 \%$; Sheba Medical Center, Israel) developed COVID-19 between 1-10 days after immunization [7]. Further, data from Tel Aviv Sourasky Medical Center indicated a $1.18 \%$ and $0.50 \%$ frequency of infection in healthcare workers after first and second doses of BNT162b2 vaccine [7]. Keehner and coworkers (2021) reported about $1 \%$ total risk of testing positive for SARS-CoV-2 for vaccinated healthcare workers from universities UCSD (San Diego, USA) and UCLA (Los Angeles, USA) [8]. This higher incidence, as compared to previous observations [3, 4], was explained with the increased routine diagnostic testing and the greater possibility of encountering sources of infection in sanitary centers [8]. However, it is interesting to note that the frequency of positives decreased over time after vaccination: from $2.5 \%$ detected at 1-7 days after the first dose to $0.16 \%$ monitored at 15 days or more after second dose [8]. Further, an increased protection in vaccinated healthcare workers was observed in data collected from St Jude Children's Research Hospital (Memphis, Tennessee), showing a frequency of $1.34 \%$ after first dose and $0.36 \%$ after second dose, suggesting that the frequencies of infected healthcare workers can differ according to vaccination status [9]. The case series of 23,234 at the University of Texas Southwestern Medical Center (UTSW) and 22,729 healthcare workers in Northern California (Stanford University) showed similar frequencies [10]. The latest study reported the presence of the B.1.427/B.1.429 variant in $36 \%$ of cases [10]. Similarly, a study conducted in the Northern Italy (Brescia, April 2021) reported a $0.57 \%$ 


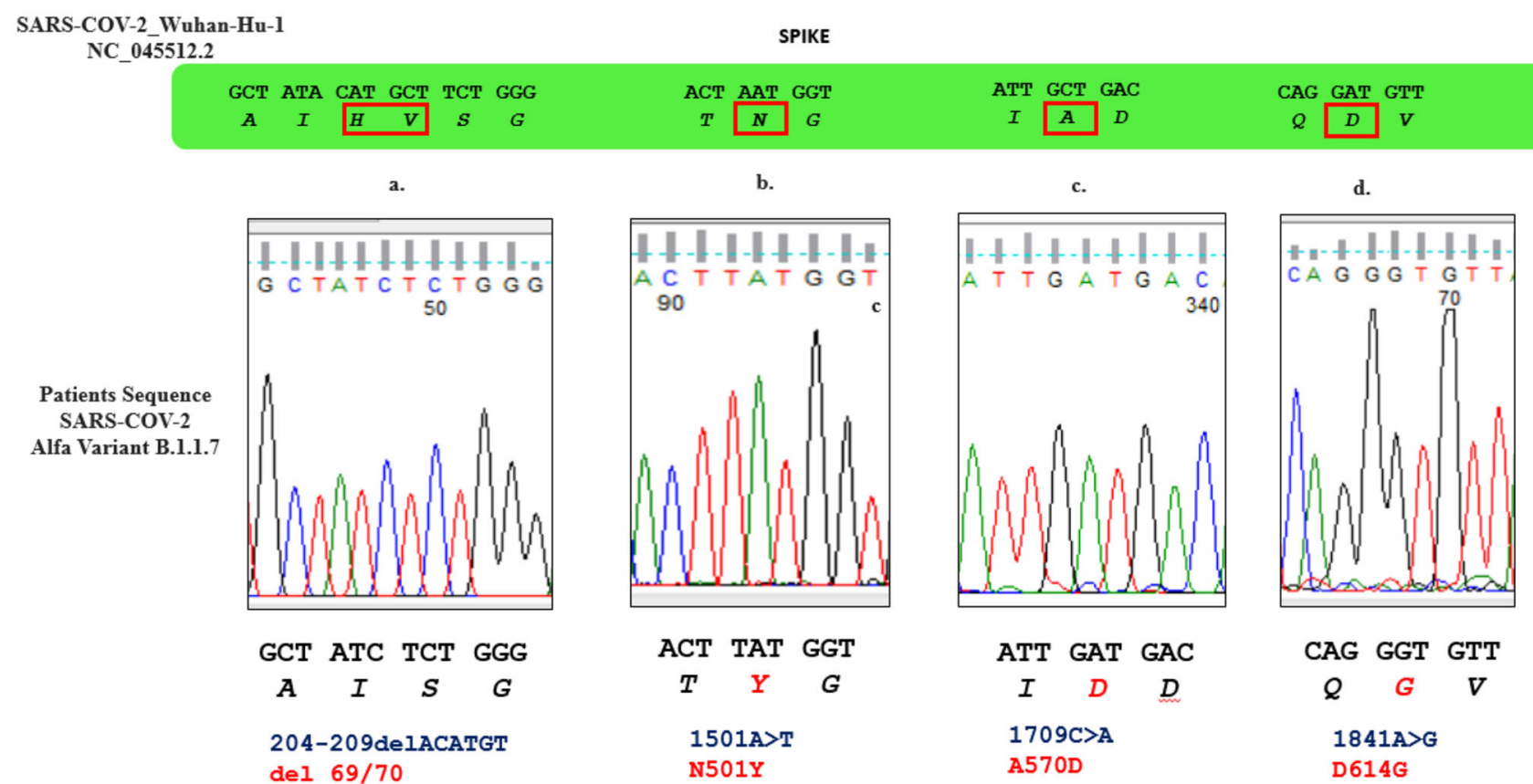

Fig. 1 Identification of SARS-CoV-2 Spike-RBD mutations using Sanger method. Sections from the electropherograms showing the 69/70, N501Y, A570D, and D614G (a., b., c., d.) associated with SARS-CoV-2 Alfa Variant B.1.1.7

frequency of infection for 6904 vaccinated healthcare workers, with a lower risk (2.6-folds) than unvaccinated colleagues but still high (6.2 folds) as compared to common population [11].

Our finding performed on swabs from 5 healthcare workers tested positive for SARS-CoV-2 highlight the B.1.1.7 variant in four cases and the B1.525 variant in one case [11]. An outbreak of infection with VOC 202,012/01lineage B.1.1.7 was previously described in two Italian physicians, one month after second dose (Southern Italy) [12].

Few studies have compared the efficacy of BNT162b2 vaccine in a population of healthcare workers including a control arm with unvaccinated subjects. To date, the SARS-CoV-2 Immunity and Reinfection Evaluation (SIREN) is the largest study from 104 UK hospitals comparing 20,641 vaccinated and 2683 unvaccinated healthcare workers [13]. During the 2-month follow-up period, 977 new infections were recorded in the unvaccinated cohort, while in the vaccinated group 71 and 9 new infections 21 days and 7 days after their first dose and second dose respectively were observed [13]. These results do not differ greatly from those reported in a study of 6493 (1090 unvaccinated and 5333 vaccinated) healthcare workers from Treviso (Italy) [14]. Although conducted with some differences in surveillance procedures and case identification, these two latter studies show good efficacy in preventing SARS-CoV-2 infection of BNT162b2 vaccine on healthcare workers with a $70-84 \%$ after 21 days from first dose and a 85-95\% after 7 days from second dose [13, 14].

From our data, it is possible to state that vaccination has substantially reduced although not eliminated the risk of SARS-CoV-2 infection in healthcare workers. Modalities and susceptibilities to infection of vaccinated subjects are at present unknown, although prolonged exposure of physicians in a COVID ward during the third wave is a major predisposing factor. The observation of high viral load value in these subjects would suggest a source of infection with high viremia, as previously described [12]. It would be considering the greater contagiousness of VOC 202,012/01-lineage B.1.1.7 variant that was first identified in Italy (Dec 2020, [15]) and widely spread throughout the territory until reaching a prevalence of $88.1 \%$ in May 2021 $[11,12]$. The different susceptibility to infection determined by genetic predisposition factors should not be overlooked [16-18].

Cases of healthcare workers infection can potentially have serious effects for transmission in working environments, including other staff members, frail or chronically ill patients, with implications not only at nosocomial level but also for entire healthcare system. Our data underline the importance of maintaining a high level of active and passive vigilance, continuing to use the specific personal protective equipment, correct distancing, and continuous laboratory screening programs.

Overall, these findings would suggest the necessity of a continuous SARS-CoV-2 surveillance even in the presence 
of full vaccination, to guarantee safety and healthiness of workers and patients [19].

Acknowledgments AM thanks to the Italian Ministry of Health and Fondazione Roma (Italy).

Author contribution CC, RP, and AM analyzed the global data and drafted the manuscript; CC, RP, AR, and AM critically revised the manuscript; All authors either performed the assays or analyzed the data of their own laboratory; All authors approved the final version of the manuscript.

Funding Not applicable.

Data availability and material Raw data provided by each laboratory will be made available at reasonable request.

Code availability All software used for this study is mentioned in the text.

\section{Declarations}

Conflict of interest The authors declare no competing interest.

Consent to participate Healthcare workers were asked to join the study protocol by signing the informed consent.

Consent for publication All authors consent to publish.

Ethical approval The protocol was approved by the Azienda Sanitaria Locale Brindisi Ethical Committee, Brindisi, Italy (Prot n. R.CE 30/21).

Open Access This article is licensed under a Creative Commons Attribution 4.0 International License, which permits use, sharing, adaptation, distribution and reproduction in any medium or format, as long as you give appropriate credit to the original author(s) and the source, provide a link to the Creative Commons licence, and indicate if changes were made. The images or other third party material in this article are included in the article's Creative Commons licence, unless indicated otherwise in a credit line to the material. If material is not included in the article's Creative Commons licence and your intended use is not permitted by statutory regulation or exceeds the permitted use, you will need to obtain permission directly from the copyright holder. To view a copy of this licence, visit http://creativecommons.org/licenses/by/4.0/.

\section{References}

1. Huang C, Wang Y, Li X, Ren L, Zhao J, Hu Y, Zhang L, Fan G, Xu J, Gu X, Cheng Z, Yu T, Xia J, Wei Y, Wu W, Xie X, Yin W, Li H, Liu M, Xiao Y, Gao H, Guo L, Xie J, Wang G, Jiang R, Gao Z, Jin Q, Wang J, Cao B (2020) Clinical features of patients infected with 2019 novel coronavirus in Wuhan, China. Lancet 395(10223):497-506. https://doi.org/10.1016/S0140-6736(20)30183-5

2. Li Q, Guan X, Wu P, Wang X, Zhou L, Tong Y, Ren R, Leung KSM, Lau EHY, Wong JY, Xing X, Xiang N, Wu Y, Li C, Chen Q, Li D, Liu T, Zhao J, Liu M, Tu W, Chen C, Jin L, Yang R, Wang Q, Zhou S, Wang R, Liu H, Luo Y, Liu Y, Shao G, Li H, Tao Z, Yang Y, Deng Z, Liu B, Ma Z, Zhang Y, Shi G, Lam TTY, Wu JT, Gao GF, Cowling BJ, Yang B, Leung GM, Feng Z
(2020) Early transmission dynamics in Wuhan, China, of novel coronavirus-infected pneumonia. N Engl J Med 382(13):1199-1207. https://doi.org/10.1056/NEJMoa2001316

3. Polack FP, Thomas SJ, Kitchin N, Absalon J, Gurtman A, Lockhart S, Perez JL, Pérez Marc G, Moreira ED, Zerbini C, Bailey R, Swanson KA, Roychoudhury S, Koury K, Li P, Kalina WV, Cooper D, Frenck RW Jr, Hammitt LL, Türeci Ö, Nell H, Schaefer A, Ünal S, Tresnan DB, Mather S, Dormitzer PR, Şahin U, Jansen KU, Gruber WC, C4591001 Clinical Trial Group (2021) Safety and Efficacy of the BNT162b2 mRNA Covid-19 Vaccine. N Engl J Med 383:2603-2615. https://doi.org/10. 1056/NEJMoa2034577

4. Dagan N, Barda N, Kepten E, Miron O, Perchik S, Katz MA, Hernán MA, Lipsitch M, Reis B, Balicer RD (2021) BNT162b2 mRNA Covid-19 vaccine in a nationwide mass vaccination setting. N Engl J Med 384(15):1412-1423. https://doi.org/10.10 56/NEJMoa2101765

5. Butt AA, Khan T, Yan P, Shaikh OS, Omer SB, Mayr F (2021) Rate and risk factors for breakthrough SARS-CoV-2 infection after vaccination. J Infect 83(2):237-279. https://doi.org/10.101 6/j.jinf.2021.05.021

6. Amit S, Regev-Yochay G, Afek A, Kreiss Y, Leshem E (2021) Early rate reductions of SARS-CoV-2 infection and COVID-19 in BNT162b2 vaccine recipients. Lancet 397(10277):875-877. https://doi.org/10.1016/S0140-6736(21)00448-7

7. Amit S, Beni SA, Biber A, Grinberg A, Leshem E, RegevYochay G (2021) Postvaccination COVID-19 among health care workers. Israel Emerg Infect Dis 27(4):1220-1222. https://doi. org/10.3201/eid2704.210016

8. Keehner J, Horton LE, Pfeffer MA, Longhurst CA, Schooley RT, Currier JS, Abeles SR, Torriani FJ (2021) SARS-CoV-2 infection after vaccination in health care workers in California. N Engl J Med 384(18):1774-1775. https://doi.org/10.1056/NEJMc2 101927

9. Tang L, Hijano DR, Gaur AH, Geiger TL, Neufeld EJ, Hoffman JM, Hayden RT (2021) Asymptomatic and symptomatic SARSCoV-2 infections after BNT162b2 vaccination in a routinely screened workforce. JAMA 325(24):2500-2502. https://doi. org/10.1001/jama.2021.6564

10. Jacobson KB, Pinsky BA, Montez Rath ME, Wang H, Miller JA, Skhiri M, Shepard J, Mathew R, Lee G, Bohman B, Parsonnet J, Holubar M (2021) Post-vaccination SARS-CoV-2 infections and incidence of presumptive B.1.427/B1.429 variant among health care personnel at a northern California academic medical center. Clin Infect Dis. https://doi.org/10.1093/cid/ciab554

11. Sansone E, Tiraboschi M, Sala E, Albini E, Lombardo M, Castelli F, De Palma G (2021) Effectiveness of BNT162b2 vaccine against the B.1.1.7 variant of SARS-CoV-2 among health care workers in Brescia, Italy. J Infect 83(1):17-18. https://doi.org/ 10.1016/j.jinf.2021.04.038

12. Loconsole D, Sallustio A, Accogli M, Leaci A, Sanguedolce A, Parisi A, Chironna M (2021) Investigation of an outbreak of symptomatic SARS-CoV-2 VOC 202012/01-lineage B.1.1.7 infection in health care workers, Italy. Clin Microbiol Infect. https://doi.org/10.1016/j.cmi.2021.05.007

13. Zhang Y, Zeng G, Pan H, Li C, Hu Y, Chu K, Han W, Chen Z, Tang R, Yin W, Chen X (2021) Safety, tolerability, and immunogenicity of an inactivated SARS-CoV-2 vaccine in healthy adults aged 18-59 years: a randomised, double-blind, placebo-controlled, phase $1 / 2$ clinical trial. Lancet Infect Dis 21(2):181-192. https://doi.org/10.1016/S0140-6736(21)00790-X

14. Fabiani M, Ramigni M, Gobbetto V, Mateo-Urdiales A, Pezzotti P, Piovesan C (2021) Effectiveness of the Comirnaty (BNT162b2, BioNTech/Pfizer) vaccine in preventing SARSCoV-2 infection among health care workers, Treviso province, Veneto region, Italy, 27 December 2020 to 24 March 2021. Euro 
Surveill 26(17):2100420. https://doi.org/10.2807/1560-7917.E S.2021.26.17.2100420

15. Lo Menzo S, Marinello S, Biasin M, Terregino C, Franchin E, Crisanti A, Cattelan A (2021) The first familial cluster of the B.1.1.7 variant of SARS-CoV-2 in the northeast of Italy. Infection 10:1-5. https://doi.org/10.1007/s15010-021-01609-6

16. Martínez-Sanz J, Jiménez D, Martínez-Campelo L, Cruz R, Vizcarra P, Sánchez-Conde M, Ron R, Rodríguez M, Herrera S, Moreno S, López-Huertas MR, Serrano-Villar S (2021) Role of ACE2 genetic polymorphisms in susceptibility to SARS-CoV-2 among highly exposed but non infected health care workers. Emerg Microbes Infect 10(1):493-496. https://doi.org/10.10 80/22221751.2021.1902755

17. Cafiero C, Rosapepe F, Palmirotta R, Re A, Ottaiano MP, Benincasa G, Perone R, Varriale E, D’Amato G, Cacciamani A, Micera A, Pisconti S (2021) Angiotensin system polymorphisms' in SARS-CoV-2 positive patients: assessment between symptomatic and asymptomatic patients: a pilot study. Pharmgen Pers Med 14:621-629. https://doi.org/10.2147/PGPM.S303666

18. Cafiero C, Re A, Micera A, Palmirotta R, Monaco D, Romano F, Fabrizio C, Di Francia R, Cacciamani A, Surico PL, D'Amato G, Pisconti S (2020) Pharmacogenomics and pharmacogenetics. in silico prediction of drug effects in treatments for novel coronavirus SARS-CoV2 disease. Pharmgen Pers Med 13:463-484. https://doi.org/10.2147/PGPM.S270069

19. Kamboj VP (2021) COVID-19 vaccines: speedy development and their use to be saviour of humanity. Natl Acad Sci Lett. https://doi.org/10.1007/s40009-021-01080-9

Publisher's Note Springer Nature remains neutral with regard to jurisdictional claims in published maps and institutional affiliations. 